\title{
Role of androgens in dhea-induced rack1 expression and cytokine modulation in monocytes
}

\author{
Emanuela Corsini ${ }^{1}$, Valentina Galbiati ${ }^{1}$, Angela Papale ${ }^{1}$, Elena Kummer ${ }^{1}$, Antonella Pinto ${ }^{2}$, Melania M. Serafini ${ }^{2}$, \\ Antonio Guaita ${ }^{3}$, Roberto Spezzano ${ }^{4}$, Donatella Caruso ${ }^{4}$, Marina Marinovich ${ }^{1}$ and Marco Racchi ${ }^{2 *}$
}

\begin{abstract}
Background: Over the past fifteen years, we have demonstrated that cortisol and dehydroepiandrosterone (DHEA) have opposite effects on the regulation of protein kinase C (PKC) activity in the context of the immune system. The anti-glucocorticoid effect of DHEA is also related to the regulation of splicing of the glucocorticoid receptor (GR), promoting the expression of GRß isoform, which acts as a negative dominant form on GRa activity. Moreover, it is very well known that DHEA can be metabolized to androgens like testosterone, dihydrotestosterone (DHT), and its metabolites $3 a-d i o l$ and $3 \beta$-diol, which exert their function through the binding of the androgen receptor (AR). Based on this knowledge, and on early observation that castrated animals show results similar to those observed in old animals, the purpose of this study is to investigate the role of androgens and the androgen receptor (AR) in DHEA-induced expression of the PKC signaling molecule RACK1 (Receptor for Activated C Kinase 1) and cytokine production in monocytes.
\end{abstract}

Results: Here we demonstrated the ability of the anti-androgen molecule, flutamide, to counteract the stimulatory effects of DHEA on RACK1 and GRß expression, and cytokine production. In both THP-1 cells and human peripheral blood mononuclear cells (PBMC), flutamide blocked the effects of DHEA, suggesting a role of the AR in these effects. As DHEA is not considered a direct AR agonist, we investigated the metabolism of DHEA in THP-1 cells. We evaluated the ability of testosterone, DHT, and androstenedione to induce RACK1 expression and cytokine production. In analogy to DHEA, an increase in RACK1 expression and in LPS-induced IL-8 and TNF-a production was observed after treatment with these selected androgens. Finally, the silencing of AR with siRNA completely prevented DHEA-induced RACK1 mRNA expression, supporting the idea that AR is involved in DHEA effects.

Conclusions: We demonstrated that the conversion of DHEA to active androgens, which act via AR, is a key mechanism in the effect of DHEA on RACK1 expression and monocyte activation. This data supports the existence of a complex hormonal balance in the control of immune modulation, which can be further studied in the context of immunosenescence and endocrinosenescence.

Keywords: Immunosenescence, PKC, Cytokines, Androgens, Signal transduction, Monocytes

\footnotetext{
*Correspondence: racchi@unipv.it

${ }^{2}$ Department of Drug Sciences - Pharmacology Unit, University of Pavia, Viale

Taramelli 14, Pavia 27100, Italy

Full list of author information is available at the end of the article
} 


\section{Background}

In early studies aimed to characterize the molecular mechanisms underlying immunosenescence, we identified a defective protein kinase C (PKC) activation central to the function of immune cells. In particular, we demonstrated that the failing element in PKC activation was the reduced expression of its scaffold protein Receptor for Activated C Kinase 1 (RACK1), which underlies the functional impairment of immune system associated with aging [1-5]. RACK1 is a $36-\mathrm{kDa}$ protein that contains seven WD-domain motifs and is related to $G$ protein $\beta$ subunits $[6,7]$. RACK1 is a highly conserved intracellular adaptor protein, which was originally identified as the anchoring protein for activated PKC $[8,9]$. In the past 20 years, the number of binding partners and validated cellular functions for RACK1 has increased. It is believed to act as signal integrator, which interconnects distinct signaling pathways to control many essential cellular processes, including protein translation, developmental processes, multiple hormonal responses, pathogen infection resistance, environmental stress responses, and miRNA production. These multiple functional roles are fitting, considering the scaffolding nature of RACK1 protein [10-14].

Relative to PKC, RACK1 is able to interact preferentially with PKC $\beta \mathrm{II}$ [8] and PKCe [9]. RACK1 stabilizes their active conformation and promotes their translocation to specific substrates in order to activate defined pathways $[6,7]$. Using antisense oligonucleotides to decrease RACK1 expression, or using a PKC $\beta$ pseudosubstrate to selectively inhibit PKC $\beta$ activation, a significant reduction in LPS-induced monocytes/macrophages activation was observed, indicating the involvement of RACK1 and $\mathrm{PKC} \beta$ in the signal transduction pathways triggered by LPS $[1,15]$.

The role of hormones in the control of RACK1 expression was suggested by a series of experimental evidence. While in old rats there is evidence that RACK1 expression and LPS-stimulated production of tumor necrosis factor- $\alpha$ (TNF $\alpha)$ is reduced in alveolar macrophages, we demonstrated that castration of young male rats produced effects on alveolar macrophages similar to those of aging. Conversely, the supplementation of dehydroepiandrosterone (DHEA) to old rats restored the age-decreased level of RACK1, LPS-stimulated production of TNF $\alpha$ in alveolar macrophages, and mitogen-induced splenocyte proliferation, suggesting that immunosenescence is partially under hormonal control and can be restored by appropriate replacement therapy [2].

Similarly, we also observed a direct correlation between circulating DHEA and RACK1 expression in human peripheral blood leukocytes. In vitro treatment with physiological concentrations of DHEA resulted in increased RACK1 expression in leukocytes and lymphocyte proliferation, confirming the role of this hormone in the modulation of RACK1 expression and immune functions [3].

DHEA is the most abundantly secreted adrenal steroid in humans. It is known to increase throughout childhood and puberty, and then to decrease with old age. The average serum DHEA concentration in men aged $25-34$ is $15.9+6.1 \mathrm{nM}$ but falls to $5.4+1.7 \mathrm{nM}$ in those aged 75-85 [16]. Reduced secretion of DHEA during aging has been related to a series of age-associated conditions, including atherosclerosis and cardiovascular disease, breast cancer, obesity, loss of muscle mass, and diabetes; there is a view that supplementation with DHEA may have a number of significant clinical uses [17].

It is well documented that DHEA affects multiple cellular functions of the endocrine, immune, and nervous systems. However, despite intense effort by scientists to elucidate the multifunction of DHEA, its mechanism of action is still elusive. Most of its physiological actions have been attributed to its conversion to either androgens or estrogens. Depending on the tissue, DHEA can be indeed metabolized to 4-androstenedione, $5 \alpha$ androstenedione, testosterone, estrogen, and other biologically active steroids $[18,19]$.

More recently, we demonstrated in THP-1 cells that DHEA can antagonize physiological functions of endogenous glucocorticoid [20] inducing a dose-related up-regulation of GR $\beta$, through a modulation in the expression of the splicing-related protein SRp30c. Moreover, GR $\beta$ knockdown prevented DHEA-induced RACK1 expression and modulation of cytokine release [21]. This data indicated the ability of GR $\beta$ to act as a dominant-negative inhibitor of GR $\alpha$ function, which could underlie the anti-glucocorticoid effect of DHEA on RACK1 protein expression.

Glucocorticoid, progestin, and androgen hormones function via their specific receptors, which recognize a common DNA binding site: the glucocorticoid response element (GRE) or hormone response element (HRE). HRE can function as a response element for all steroid classes except for estrogens, allowing extensive crosstalk between receptors and shared target genes [22, 23]. To shed light on the mechanisms underlying DHEAinduced RACK1 expression and cytokine production in monocytes, we investigated the role of androgens and androgen receptor on DHEA effects. Collectively, our findings demonstrate that, similarly to DHEA, other androgens exerted stimulatory effects on immune cells, and AR silencing completely prevented DHEA-induced RACK1, supporting the notion that DHEA conversion to androgens and AR are central to the biological effects of DHEA in monocytes. This data further contributes to our understanding of the mechanism of action of DHEA. 


\section{Methods}

\section{Chemicals}

Four-Androstene-3, 17-dione (androstenedione), DHEA, DHT, 3 $\alpha$-DIOL, 3 $\beta$-DIOL, flutamide (Flut), testosterone were obtained from Sigma Aldrich (St Louis, MO, USA). Steroids were dissolved in dimethyl sulfoxide (DMSO) at $50 \mathrm{mM}$ and further diluted (the final concentration of DMSO in culture medium was $0.2 \%$ ). Lipopolysaccharide from Escherichia coli serotype 0127:B8 (LPS) was from Sigma Aldrich. All cell culture reagents and supplements were from Sigma. Antibodies against RACK1 and AR were from BD Biosciences (Franklin Lakes, NJ, USA), GR $\beta$ from Abcam (Cambridge, UK), and anti-human $\beta$-actin from Sigma Aldrich. AR siRNA and scrambled controls were from Cell Signaling Technology (Danvers, MA, USA). Electrophoresis reagents were from Bio-Rad (Hercules, CA, USA). All reagents were purchased at the highest purity available.

\section{Cells}

THP-1 cells, obtained from Istituto Zooprofilattico di Brescia (Italy), were diluted to $10^{6}$ cells $/ \mathrm{mL}$ in RPMI 1640 without phenol red containing $2 \mathrm{mM} \mathrm{L}$-glutamine, $0.1 \mathrm{mg} / \mathrm{mL}$ streptomycin, $100 \mathrm{IU} / \mathrm{mL}$ penicillin, gentamycin $10 \mu \mathrm{g} / \mathrm{ml}, 50 \mu \mathrm{M} 2$-mercaptoethanol, supplemented with $5 \%$ heated-inactivated dialyzed fetal calf serum (culture media) and cultured in suspension at $37{ }^{\circ} \mathrm{C}$ in $5 \%$ $\mathrm{CO}_{2}$ incubator. Cells were treated as reported in the legend to figures.

\section{Human volunteers and ethical statement}

Peripheral blood mononuclear cells (PBMCs) were isolated by Ficoll gradient centrifugation from fresh blood of human volunteers. Five healthy females (23-52 yearold) were selected according to the guidelines of the Italian Health authorities, and to the Declaration of Helsinki principles, within a protocol approved by the Ethics Committee of the University of Pavia (NCT01345110), and signed an informed consent. Following centrifugation at $900 \mathrm{~g}$ at $25^{\circ} \mathrm{C}$ for $30 \mathrm{~min}$ with no brake, the PBMCs layer was removed and washed twice in Hanks' balanced salt solution (HBSS). PBMCs were resuspended at $10^{6} / \mathrm{ml}$ in culture media and treated as reported in the legend to figures.

\section{Cell viability}

Cytotoxicity was assessed by leakage of lactate dehydrogenase (LDH). LDH activity was determined in cellfree supernatants using a commercially available kit (Takara Bio Inc., Japan). Results are expressed as optical density (OD).

\section{Real time RT-PCR}

Total RNA was isolated at different times of treatment using a commercially available kit (TriReagent from Sigma) following the supplier's instructions. For the synthesis of cDNA, $2.0 \mu \mathrm{g}$ of total RNA was retro-transcribed using the High-Capacity cDNA Archive Kit from Applied Biosystems (Foster City, CA, USA) following the supplier's instructions. RACK1 gene expression was evaluated by real-time reverse transcription-polymerase chain reaction (Real-time PCR). For PCR-analysis, Taq-ManTM-PCR technology was used. PCRs were performed in duplicate and according to the standard protocol suggested by the manufacturer. For each PCR reaction, $10 \mathrm{ng}$ of total RNA were used. The $18 \mathrm{~S}$ ribosomal RNA transcription was used as endogenous reference, and the quantification of the transcripts was performed by the $2^{-\Delta \Delta C T}$ method [24].

\section{Western blot analysis}

The presence of RACK1 and AR in cell homogenates was assessed by Western blot analysis. Briefly, cells $\left(3 \times 10^{6}\right)$ were treated as described in the legends. At the end of treatments, cells were collected, washed once with PBS, centrifuged and lysed in $100 \mu \mathrm{l}$ of homogenization buffer (50 mM TRIS, $150 \mathrm{mM} \mathrm{NaCl,} 5$ mM EDTA pH 7.5, $0.5 \%$ Triton X-100, $50 \mu \mathrm{M}$ PMSF, $2 \mu \mathrm{g} / \mathrm{mL}$ aprotinin, $1 \mu \mathrm{g} / \mathrm{mL}$ pepstatin and $1 \mu \mathrm{g} / \mathrm{mL}$ leupeptin) and denatured for $10 \mathrm{~min}$ at $100{ }^{\circ} \mathrm{C}$. The protein content of the cell lysate was measured using a commercial kit (Bio-Rad). $5 \mu \mathrm{g}$ (for RACK1) or $10 \mu \mathrm{g}$ (for GR $\beta$ and AR) of extracted proteins were electrophoresed in a $12 \%$ SDS-polyacrylamide gel under reducing conditions. The proteins were then transferred to PVDF membrane (Amersham, Little Chalfont, UK). The proteins were visualized using primary antibodies for RACK1 (1:1000), GR $\beta$ (1:1000), AR (1:1000) and $\beta$-actin (1:5000) and developed using enhanced chemiluminescence (Pierce, Thermo Scientific, Rockford, IL, USA). The image of the blot was acquired and analyzed with the Molecular Imager Gel Doc XR (BioRad).

\section{Cytokine production}

Cytokine production was assessed in cell free supernatants by specific sandwich ELISAs, commercially available (R\&D System, Minneapolis, MN, USA). Cell-free supernatants obtained by centrifugation at $1200 \mathrm{rpm}$ for $5 \mathrm{~min}$ were stored at $-20{ }^{\circ} \mathrm{C}$ until measurement. Limits of detection were $15 \mathrm{pg} / \mathrm{ml}$. Results are expressed in $\mathrm{pg} / \mathrm{ml}$.

\section{Quantitative analysis of steroids by LC-MS/MS}

Intracellular testosterone and its metabolites DHT, $3 \alpha-$ DIOL and $3 \beta-D I O L$ were assessed by LC-MS/MS as previously described [25]. Cells $\left(1 \times 10^{7}\right)$ were treated with DHEA $10 \mathrm{nM}$ or DMSO as vehicle control for $1 \mathrm{~h}$, washed twice with PBS and lysed. Samples, added with $13 \mathrm{C}$ internal standards, were extracted and purified with 
$1 \mathrm{ml}$ of $\mathrm{MeOH} /$ acetic acid (99:1, v/v). The organic phases were passed through an SPE cartridge, steroids fraction concentrated and transferred into auto-sampler vials before LC-MS/MS analysis. Quantitative analysis was performed on the basis of calibration curves prepared and analyzed as described above for samples. Positive atmospheric pressure chemical ionization (APCI) experiments were performed using a linear ion trap-mass spectrometer (LTQ, ThermoElectron Co., San Jose, CA, USA) and the LC mobile phases were (A) $\mathrm{H} 2 \mathrm{O} / 0.1 \%$ formic acid and (B) methanol $(\mathrm{MeOH}) / 0.1 \%$ formic acid. The gradient (flow rate $0.5 \mathrm{ml} / \mathrm{min}$ ) was described previously [25].

\section{Small interference RNA (siRNA) for AR}

To evaluate the role of AR in DHEA-induced RACK1 expression, silencing experiments were conducted. The effect of inducing RNA interference on AR was assessed using commercially available reagents (SignalSilence ${ }^{\mathrm{Tn}}$ siRNA from Cell Signaling Technology) following the manufacturer's instructions. As control, siRNA (scr), an siRNA sequence that will not cause the specific degradation of any cellular messages was used. Forty-eight hours after siRNA transfection, Western blot analysis using AR antibody was performed to confirm silencing in whole cell lysates. Cells were then adjusted to $10^{6} / \mathrm{ml}$ and treated with DHEA (10 nM) or DMSO as vehicle control for $16 \mathrm{~h}$ to assess RACK1 mRNA expression and $24 \mathrm{~h}$ to assess RACK1 protein expression.

\section{Statistical analysis}

All experiments were performed at least three times, with representative results shown. Data are expressed as mean \pm standard deviation (SD). Statistical analysis was performed using GraphPad InStat version 3.0a for Macintosh (GraphPad Software, San Diego, CA, USA). Differences were considered significant at $p \leq 0.05$.

\section{Results}

The anti-androgen flutamide prevents the stimulatory effects of DHEA on RACK1 expression and cytokine production

The aim of this study was to investigate the role of androgens and AR in the immunostimulatory effects of DHEA. To address this question, we first investigated the ability of the anti-androgen flutamide to modulate the stimulatory effects of DHEA in both the human promyelocytic THP-1 cell line, and in human peripheral blood mononuclear cells (PBMCs). THP-1 is a human leukemia monocytic cell line derived from the peripheral blood of a 1-year-old human male with acute monocytic leukemia. This cell line has been extensively used to study monocyte/macrophage functions, mechanisms, and signaling pathways [26]. PBMCs were obtained from five adult healthy females (28-year-old) and were used to support the relevance of data obtained in THP-1 cells and to exclude possible gender effects. THP-1 cells and PBMCs were treated for $1 \mathrm{~h}$ with flutamide $(50 \mu \mathrm{M})$, then a physiologically relevant concentration of DHEA (10 nM) or DMSO as vehicle control was added. After $16 \mathrm{~h}$ of treatment, Real Time-PCR was used to assess RACK1 mRNA expression. In both experimental models, flutamide completely prevented DHEA-induced RACK1 mRNA expression (Fig. 1a and b), suggesting the role of $A R$ in the effect of DHEA on RACK1 expression.

The rest of the data presented was conducted using THP-1 cells. Next, the effect of flutamide on DHEAinduced RACK1 protein expression and cytokine production was investigated. Cells were treated with DHEA for $24 \mathrm{~h}$ as optimal time for RACK1 protein expression [27]. After DHEA treatment, LPS $(10 \mathrm{ng} / \mathrm{ml})$ was added for 3 and $24 \mathrm{~h}$ to assess TNF- $\alpha$ and IL- 8 release, respectively. Flutamide not only prevented DHEA-induced RACK1 mRNA expression, but also RACK1 protein expression as assessed by Western blot analysis (Fig. 1c), and cytokine production as assessed by LPS $(10 \mathrm{ng} / \mathrm{ml})$ induced TNF- $\alpha$ and IL-8 release (Fig. 1e and f). Similar inhibitory effects on LPS-induced cytokine production were observed in human PBMC treated with DHEA in the presence of flutamide (data not shown). The release of TNF- $\alpha$ and IL-8 in DMSO treated cells was below the detection limit of the ELISA (data not shown). The inhibitory effect of flutamide was not due to cytotoxicity, as assessed by identical LDH leakage in control and treated cells: in control cells the optical density (OD) value was $0.582 \pm 0.023$ vs $0.597 \pm 0.017$ in flutamide treated cells.

Furthermore, considering the anti-glucocorticoid effect of DHEA, mediated by the induction of GR $\beta$ [21], we demonstrate here that flutamide also prevents DHEAinduced GR $\beta$ protein expression (Fig. 1d). This data clearly indicates that AR is central to the action of DHEA, and confirms early data obtained in experimental animals on the role of androgens in the maintenance of RACK1 expression and immune function [2].

\section{DHEA is metabolized to androgens in THP-1 cells, and other androgens have a similar effect to that of DHEA} Most of DHEA physiological actions have been attributed to its conversion to either androgens or estrogens, therefore, we investigated the ability of THP-1 cells to metabolize DHEA. THP-1 cells were treated for $1 \mathrm{~h}$ with DHEA (10 nM) or DMSO as vehicle control. Testosterone, DHT, and its metabolites $3 \alpha$-diol and $3 \beta$-diol were evaluated by LC-MS/MS in cell lysates (Fig. 2a). Data indicates the ability of THP-1 cells to rapidly convert DHEA to androgens. This finding prompted us to investigate the ability of the androgens testosterone, DHT, 
A

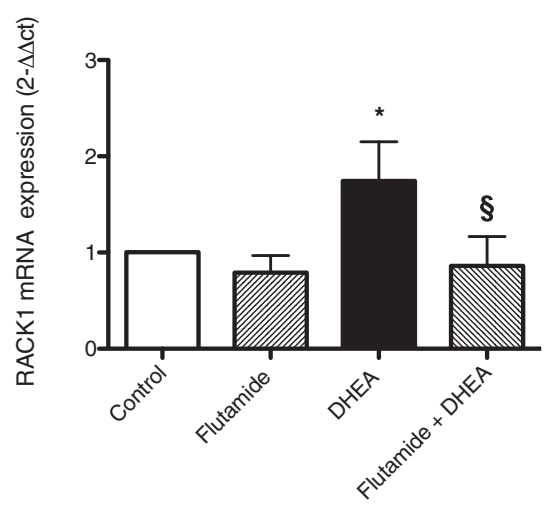

C

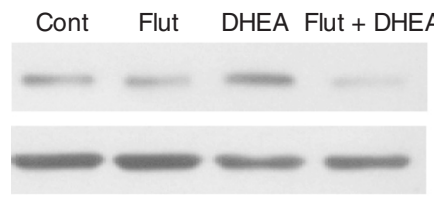

E

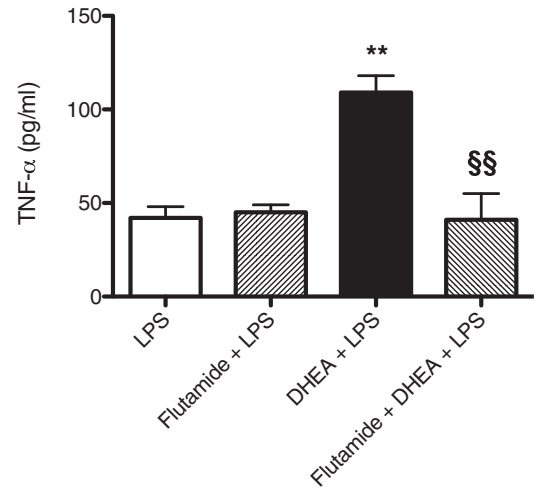

B

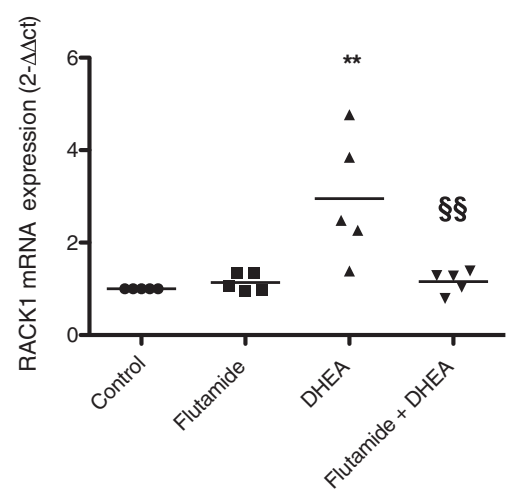

D

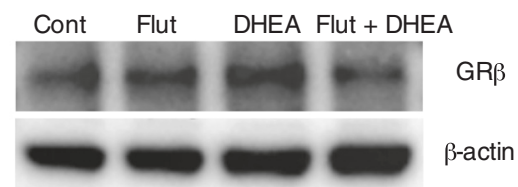

$\mathbf{F}$

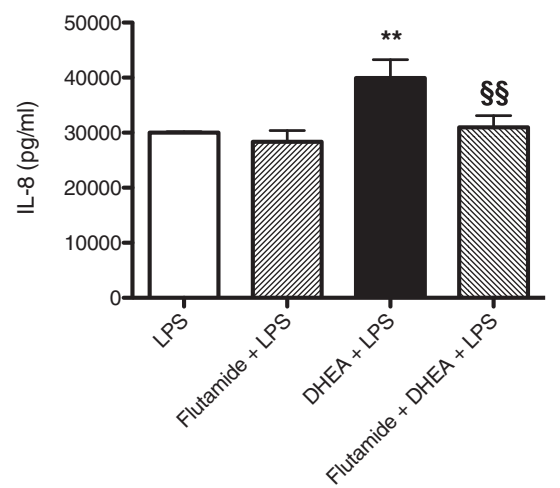

Fig. 1 The anti-androgen flutamide prevents DHEA-induced effects. a, b RACK1 mRNA expression. THP-1 cells (a) or PBMC (b) were treated with flutamide $(50 \mu \mathrm{M})$ or DMSO for $1 \mathrm{~h}$, followed by DHEA (10 nM) or DMSO for $16 \mathrm{~h}$. DMSO (0.1 \% final concentration) was used as vehicle control in all experiments. RACK1 mRNA expression was assessed by Real Time-PCR. Results are expressed as mean \pm SD of three independent experiments (a) or as dots of individual donors responses (b). ${ }^{*} p<0.05$ vs Control cells and $\$ p<0.05$ vs DHEA alone. c, d Representative Western blot analyses of RACK1 (c) or GRß (d) immunoreactivity in THP-1 cells. $\beta$-Actin immunoreactivity was used as protein loading control. Representative Western blots are reported. Cells were treated with flutamide (Flut, $50 \mu \mathrm{M}$ ) or DMSO (Cont) for $1 \mathrm{~h}$ and then DHEA (10 nM) or DMSO was added for $24 \mathrm{~h}$. e, $\mathbf{f}$ Effect on cytokine production. THP-1 cells were treated with flutamide $(50 \mu \mathrm{M})$ for DMSO or $1 \mathrm{~h}$, and then DHEA (10 nM) or DMSO was added. After $24 \mathrm{~h}$, LPS (10 ng/ml) was added for $3 \mathrm{~h}$ to assess TNF-a release (e) or $24 \mathrm{~h}$ to assess IL-8 release (f). Results are expressed as mean \pm SD, $n=3$ replicates. Data is representative of three independent experiments. Statistical analysis was performed with Tukey's multiple comparison test with ${ }^{* *} p<0.01$ vs LPS treated cells and $\S \S, p<0.01$ vs DHEA + LPS treated cells

and 4-androstenedione, the first intermediate of DHEA in the synthesis of testosterone, to induce RACK1 expression and cytokine production. In analogy to DHEA, an increase in RACK1 expression (both mRNA and protein) and in LPS-induced IL- 8 and TNF- $\alpha$ production was observed after treatment with these androgens (Figs. 2 and 3). The role of DHT in DHEA-induced RACK1 expression was also corroborated by the ability of finasteride, a $5 \alpha$-reductase inhibitor, to completely block the effect of DHEA on RACK1 mRNA expression (Fig. 2b).

In details, THP-1 cells were treated with increasing concentrations of testosterone (1-100 nM). These concentrations are physiologically relevant, as the ranges of plasma levels of testosterone in female are $0.7-3 \mathrm{nM}$, and 10-50 $\mathrm{nM}$ in male. Testosterone was able to induce a doserelated increase of RACK1 mRNA expression (Fig. 2c), and protein (Fig. $2 \mathrm{~d}$ ), which was associated with a statistically 

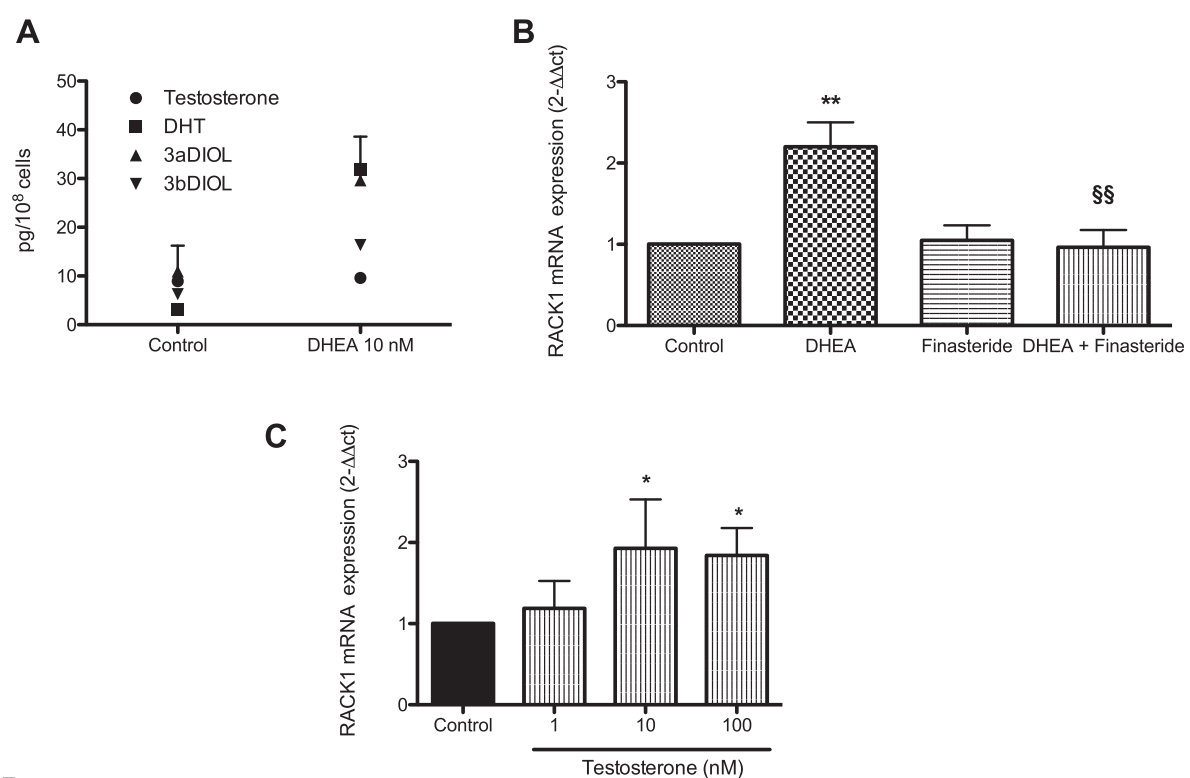

D

testosterone (nM)

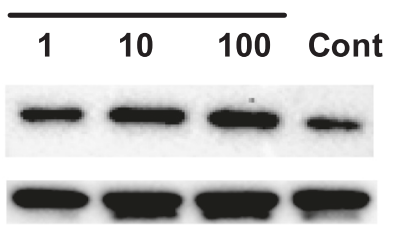

RACK1

$\beta$-actin

E

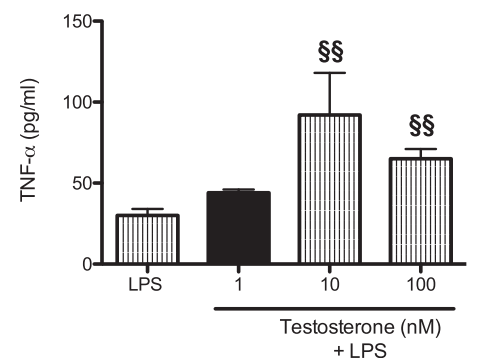

Testosterone (nM)

$\mathbf{F}$
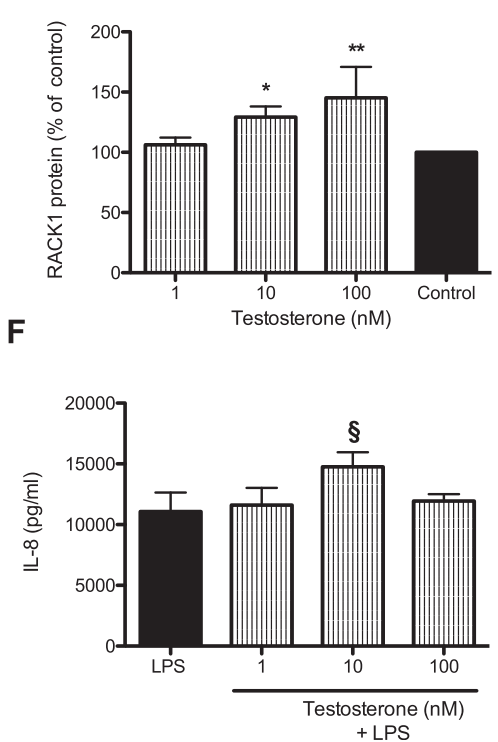

Fig. 2 DHEA is metabolized to DHT in THP-1 cells; and testosterone (similarly to DHEA) affects RACK1 expression. a DHEA metabolism. THP-1 cells $\left(10^{7}\right)$ were treated with (10 nM) or DMSO as vehicle control for $1 \mathrm{~h}$. Testosterone, DHT, and its metabolites $3 a$-diol and $3 \beta$-diol were assessed by LC-MS/MS in cell lysates. Each dot represents the mean \pm SD of 4 samples obtained in two independent experiments. $\mathbf{b}$ The $5 a-r e d u c t a s e$ inhibitor finasteride blocks DHEA-induced RACK1 expression. THP-1 cells were treated with finasteride $(0.1 \mu \mathrm{M})$ for $1 \mathrm{~h}$ and then DHEA (10 nM) or DMSO as vehicle control was added for $16 \mathrm{~h}$. RACK1 mRNA expression was assessed by Real Time-PCR. Results are expressed as mean \pm SD of three independent experiments, with $\$ p<0.05$ vs DHEA alone. $\mathbf{c}$, d Testosterone induces a dose-related increase in RACK1 expression. THP-1 cells were treated with increasing concentrations of testosterone (1-100 nM) or DMSO as vehicle control for $16 \mathrm{~h}$ for mRNA expression (c) or $24 \mathrm{~h}$ for protein expression (d). RACK1 mRNA expression was assessed by Real Time-PCR. Results are expressed as mean \pm SD of four independent experiments, with ${ }^{*} p<0.05$ vs Control cells. RACK1 immunoreactivity was assessed by Western blot analysis of cell lysates. $\beta$-Actin immunoreactivity was used as protein loading control and to normalize RACK1 expression. A representative Western blot is reported together with a densitometric analysis of data obtained from three independent experiments. Results are expressed as mean $\pm S D$, with ${ }^{*} p<0.05$ vs Control cells. e, f Effect of testosterone on LPS-induced cytokine production. THP-1 cells were treated with increasing concentrations of testosterone (1-100 nM) or DMSO as vehicle control. After $24 \mathrm{~h}$, LPS $(10 \mathrm{ng} / \mathrm{ml})$ was added for $3 \mathrm{~h}$ to assess TNF-a release (e) or $24 \mathrm{~h}$ to assess $\mathrm{IL}-8$ release (f). Results are expressed as mean $\pm S D, n=3$ replicates. Data is representative of three independent experiments. Statistical analysis was performed with Tukey's multiple comparison test with $\S p<0.05$ and $\S \S p<0.01$ vs LPS treated cells 
A

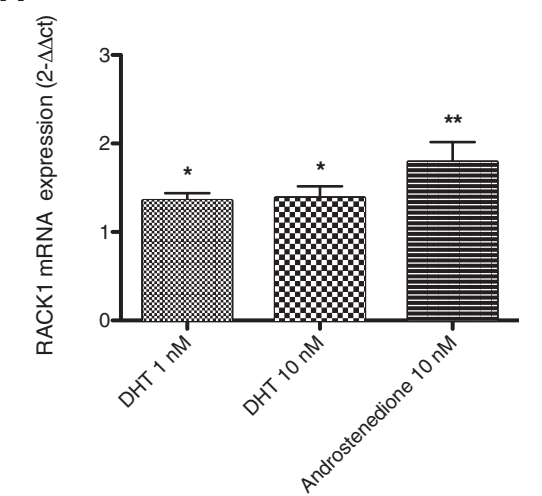

C

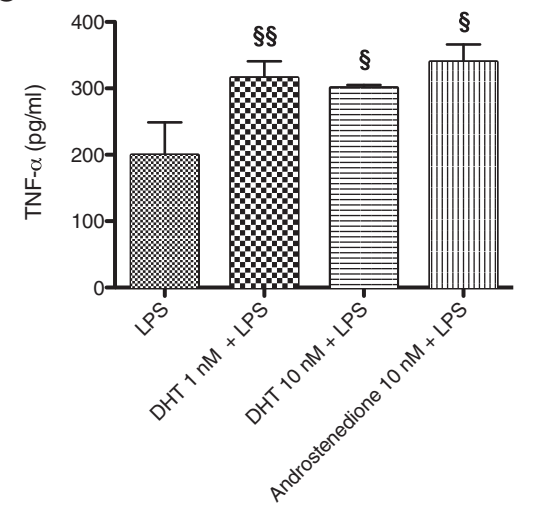

B

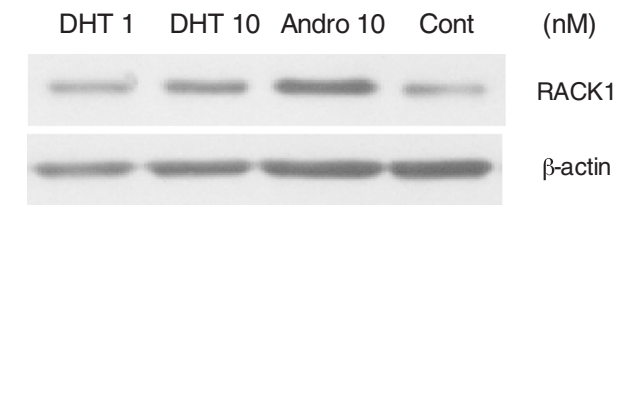

D

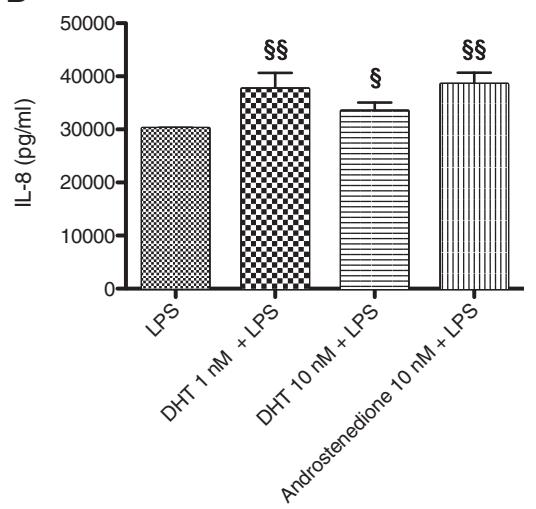

Fig. $3 \mathrm{DHT}$ and androstenedione, similarly to DHEA, affect RACK1 expression. $\mathbf{a}$, b Increased RACK1 expression following treatment with DHT and androstenedione. THP-1 cells were treated with increasing concentrations of DHT (1-10 nM), androstenedione (10 nM) or DMSO as vehicle control for $16 \mathrm{~h}$ for mRNA expression (a) or $24 \mathrm{~h}$ for protein expression (b). RACK1 mRNA expression was assessed by Real Time-PCR. Results are expressed as mean \pm SD of three independent experiments, with ${ }^{*} p<0.05$ and ${ }^{* *} p<0.01$ vs Control cells. RACK1 immunoreactivity was assessed by Western blot analysis of cell lysates $(\mathbf{b})$. A representative Western blot of RACK1 immunoreactivity in cell lysate is shown. $\mathbf{c}, \mathbf{d}$ Effect of DHT and androstenedione on LPS-induced cytokine production. THP-1 cells were treated with increasing concentrations of DHT (1-10 nM), androstenedione (10 nM) or DMSO as vehicle control. After 24 h, LPS (10 ng/ml) was added for $3 \mathrm{~h}$ to assess TNF-a release (c) or $24 \mathrm{~h}$ to assess IL-8 release (d). Results are expressed as mean $\pm S D, n=3$ replicates. Data is representative of three independent experiments. Statistical analysis was performed with Tukey's multiple comparison test with $\S p<0.05$ and $\S \S p<0.01$ vs LPS treated cells

significant increase in the response to LPS $(10 \mathrm{ng} / \mathrm{ml})$ as assessed by TNF- $\alpha$ and IL-8 release (Fig. 2e). $10 \mathrm{nM}$ testosterone was the most effective concentration.

As shown in Fig. 3, THP-1 cells were treated with DHT $(1$ and $10 \mathrm{nM})$ and 4 -androstenedione $(10 \mathrm{nM})$ for $16 \mathrm{~h}$ to assess the effect on mRNA expression (Fig. 3a), or $24 \mathrm{~h}$ for protein expression (Fig. 3b). Following $24 \mathrm{~h}$ of treatment with DHT or 4-androstenedione, LPS (10 ng/ml) was added for 3 and $24 \mathrm{~h}$ to assess TNF- $\alpha$ and IL- 8 release, respectively (Fig. 3c and d). Both DHT and 4androstenedione were able to significantly increase RACK1 mRNA and protein expression, as well as the response to LPS. Compared to testosterone, DHT appears to be more potent: at $1 \mathrm{nM}$ the increase in RACK1 expression and in LPS-induced cytokine production was already statistically significant, whereas testosterone 1 nM was ineffective (Fig. 2). This is consistent with the notion that DHT is more potent than testosterone.
Finally, to confirm that AR is necessary to mediate DHEA-induced RACK1 expression, silencing experiments were conducted. As shown in Fig. 4, AR silencing completely prevented DHEA-induced RACK1 mRNA and protein expression, supporting the notion that AR is indeed involved in DHEA effects. Additional confirmation of the link between AR and the expression of RACK1 came from the observation that the silencing of AR is associated with a reduction in RACK1 immunoreactivity, as assessed by Western blot analysis of cell lysates following $48 \mathrm{~h}$ of silencing (Fig. 4).

\section{Discussion}

DHEA has been reported to have several beneficial effects in aging humans $[17,19,28]$, and it is therefore of interest to determine the mechanisms behind these diverse effects, which are still incompletely understood. In addition, with the current utilization of DHEA as a dietary supplement, 


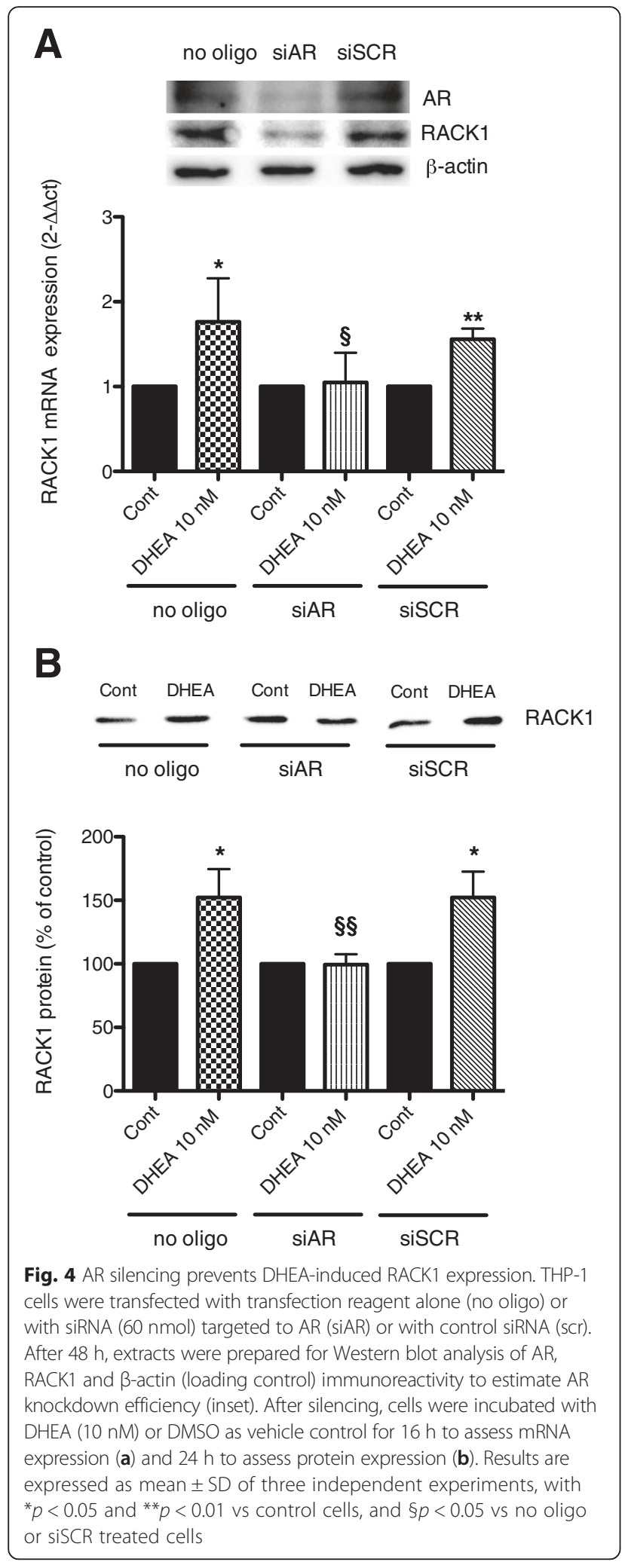

the mechanism of action of this sterol and its metabolites is important to study. The main purpose of this study was to shed light on the mechanism underlying the effect of
DHEA on RACK1 expression and on the activation of innate immune cell, as well as to reconcile data we have accumulated over the last fifteen years [2, 21]. In the present manuscript, our results indicate that the activation of AR is central to the action of DHEA on RACK1 expression and cytokine release. In THP-1 cells, we found that DHEA is rapidly converted to DHT and downstream metabolites. We demonstrated that the effect of DHEA and its metabolites on RACK1 expression could be completely prevented by using flutamide as an AR antagonist, blocking the expression of the receptor by siRNA, or using finasteride to block DHT synthesis, strongly suggesting a pivotal role of androgens and AR in RACK1 modulation. Overall, these findings contribute to our understanding of the physiological role of hormones in monocyte functions, and support the use of DHEA to manipulate monocyte activation and to counteract immunosenescence. However, the need for large, long-term placebo controlled clinical trials to demonstrate safety and efficacy of DHEA is still an open question [17].

We have previously demonstrated that cortisol and DHEA have opposite effects on the regulation of PKC activity involved in immune processes such as cytokine release and lymphocyte proliferation [2, 27]. Physiological concentrations of cortisol exert an inhibitory effect on RACK1 expression through the presence of a glucocorticoid receptor (GR)-responsive sequence on the promoter region of the human guanine nucleotide-binding protein $\beta$-2-like 1 (GNB2L1) gene, which codes for RACK1 protein [29]. Conversely, treatment with DHEA could restore the levels of RACK1 protein both in vivo and in vitro [2]. In the context of the immune system, we demonstrated that the anti-glucocorticoid effect of DHEA on RACK1 expression, and the related PKC signaling, may be due to the influence of DHEA on alternative splicing of the mRNA coding for the human GR in favor of the expression of the $\beta$ isoform [21]. In the current manuscript, we show that by blocking AR using flutamide, DHEA-induced GR $\beta$ protein expression could be completely prevented, positioning AR at the center of the action of DHEA. In both human PBMC and THP-1 cells, flutamide prevented DHEA-induced RACK1 mRNA expression and LPS-induced cytokine production. Similarly, blocking the expression of the receptor by the use of siRNA, the actions of DHEA on the same mechanisms was impaired.

DHEA mediates its action via several signaling pathways involving specific membrane receptors. These pathways and receptors include the G-coupled protein receptors demonstrated in bovine aortic endothelial cells and human umbilical vein endothelial cells, the $\sigma 1-$ receptor in neurons, and via transformation into androgen and estrogen derivatives acting through their specific receptors $[17,19,28]$. On the other hand, no 
specific DHEA responsive nuclear receptor has been identified to date; and it is believed that the majority of physiological actions of DHEA should be attributed, depending on the tissue, to its conversion to either androgens or estrogens [19]. In THP-1 cells, we found that DHEA was converted to DHT, with its downstream metabolites $3 \alpha-$ diol and $3 \beta$-diol already appearing after $1 \mathrm{~h}$ of exposure. To support the role of androgens in DHEA effects, we found that by blocking $5 \alpha$-reductase with finasteride, which prevents the conversion of testosterone to DHT as well as the conversion of 4 -androstene-3,17-dione to $5 \alpha$ androstanedione (which is then metabolized by $17 \beta-$ hydroxysteroid oxidoreductase to DHT), DHEA-induced RACK1 expression can be prevented, implicating DHT as an effector androgen. The positive modulation of RACK1 expression induced by the androgenic effect of DHEA results in an increased response to LPS, as assessed by cytokine production. Compared to testosterone, DHT appears to be a more potent stimulus. At $1 \mathrm{nM}$ DHT was already active, whereas testosterone at the same concentration was ineffective; this is in accordance with the higher stability of the DHT-AR complex [30].

Cadwallader et al. [31] have investigated hAR and hGR nuclear translocation in transiently transfected COS cells. Specific ligands induce rapid and robust nuclear translocation, without cross reactivity. For testosterone there was a slightly higher rate of transport calculated for the $50 \mathrm{nM}$ dose than the $100 \mathrm{nM}$, which may explain the slightly lower activity of testosterone $100 \mathrm{nM}$ that we observed compared to $10 \mathrm{nM}$ (Fig. 2). This could be of physiological significance, since $10 \mathrm{nM}$ is close to the physiological concentrations of androgens $(0.7-3 \mathrm{nM}$ in females and $10-50 \mathrm{nM}$ in males). The loss of the immunostimulatory effect of DHEA by blocking or silencing the $\mathrm{AR}$ is consistent with the observation that AR ablation in myeloid cells tends to establish an immunosuppressive environment [32, 33].

\section{Conclusions}

Overall, these data, together with the ability of physiologically relevant concentrations of testosterone and DHT to induce RACK1 expression, supports the notion that the metabolic transformation of DHEA to androgens and their binding to AR are required for DHEA-induced RACK1 expression and cell activation. Additional studies are required to fully understand the mechanism of AR action following DHEA treatment, especially the assessment of specific targets and/or coregulatory proteins recruited, which may also contribute to the effect of AR via interference and modulation of GR splicing isoform production, and in turn, their effects on the promoter region of GNB2L1 gene coding for RACK1 protein.

Hormones have an important role in homeostasis and function of the immune system. Sex hormones appear to have distinctive and exclusive roles in the development of the immune system and in shaping the immune responses [34]. It is intriguing to note that pharmacological concentrations of androgens have been reported to reduce immune cell activation (reviewed in ref [35]).

We identified DHEA conversion to androgens and binding to AR as necessary steps in DHEA-induced cell activation, and considering the opposite effect of cortisol, the data supports the existence of a complex hormonal balance between cortisol and androgens, which orchestrates RACK1 expression and monocyte activation, leading the way to novel therapeutic targets for immune modulation.

\section{Abbreviations \\ AR: androgen receptor; DHEA: dehydroepiandrosterone: \\ DHT: dihydrotestosterone; DMSO: dimethyl sulfoxide; GR: glucocorticoid receptor; IL: interleukin; LPS: lipopolysaccharide; PBMCs: peripheral blood mononuclear cells; RACK1: receptor for activated kinase 1; SCR: scramble: TNF: tumor necrosis factor.}

\section{Competing interests}

The authors declare that they do not have actual or potential conflict of interest including financial, personal or other relationships with other people or organizations within three years of beginning the submitted work that could inappropriately influence, or be perceived to influence, the work.

\section{Authors' contributions}

EC and MM designed the study, performed cell treatments and with MR wrote the manuscript. VG was responsible for ELISAs. AP and MMS were responsible for Western blot analysis; AP and EK performed RT-PCR. AG recruited human volunteers and collected blood; while RS performed preparation and analysis of the sample for LC-MS/MS and DC was responsible for LC-MS/MS. All authors read and approved the final manuscript.

\section{Acknowledgements}

This study was partially supported by funding from PRIN2009. The authors would like to express their thanks to Dr Nicole Simonetti for her critical review and editing in the preparation of this work.

\section{Author details}

'Laboratory of Toxicology, Department of Pharmacological and Biomolecular Sciences (DiSFeB), Università degli Studi di Milano, Milan, Italy. ${ }^{2}$ Department of Drug Sciences - Pharmacology Unit, University of Pavia, Viale Taramelli 14, Pavia 27100, Italy. ${ }^{3 " G o l g i ~ C e n c i " ~ F o u n d a t i o n, ~ A b b i a t e g r a s s o, ~ I t a l y . ~}{ }^{4}$ Mass Spectrometry Laboratory "Giovanni Galli", DiSFeB, Università degli Studi di Milano, Milan, Italy.

Received: 25 February 2016 Accepted: 10 May 2016

Published online: 29 May 2016

\section{References}

1. Corsini E, Battaini F, Lucchi L, Marinovich M, Racchi M, Govoni S, Galli CL. A defective protein kinase $C$ anchoring system underlying age-associated impairment in TNF-alpha production in rat macrophages. J Immunol. 1999; 163:3468-73.

2. Corsini E, Lucchi L, Meroni M, Racchi M, Solerte B, Fioravanti M, Viviani B, Marinovich M, Govoni S, Galli CL. In vivo dehydroepiandrosterone restores age-associated defects in the protein kinase $C$ signal transduction pathway and related functional responses. J Immunol. 2002:168:1753-8.

3. Corsini E, Racchi M, Sinforiani E, Lucchi L, Viviani B, Rovati GE, Govoni S, Galli CL, Marinovich M. Age-related decline in RACK1 expression in human leukocytes is correlated to plasma levels of dehydroepiandrosterone. J Leukoc Biol. 2005:77:247-56.

4. Racchi M, Sinforiani E, Govoni S, Marinovich M, Galli CL, Corsini E. RACK1 expression and cytokine production in leukocytes obtained from $A D$ patients. Aging Clin Exp Res. 2006;18:153-7. 
5. Corsini E, Vismara L, Lucchi L, Viviani B, Govoni S, Galli CL, Marinovich M, Racchi M. High interleukin-10 production is associated with low antibody response to influenza vaccination in the elderly. J Leukoc Biol. 2006;80:376-82.

6. McCahill A, Warwicker J, Bolger GB, Houslay MD, Yarwood SJ. The RACK1 scaffold protein: a dynamic cog in cell response mechanisms. Mol Pharmacol. 2002:62:1261-73.

7. Adams DR, Ron D, Kiely PA. RACK1, a multifaced scaffolding protein: structure and function. Cell Commun Signal. 2011;6:9-22.

8. Ron D, Chen CHSP, Caldwell J, Jamieson L, Orr E, Mochly-Rosen D. Cloning of an intracellular receptor for protein kinase C: a homolog of the beta subunit of G proteins. Proc Natl Acad Sci U S A. 1994;91:839-43.

9. Pass JM, Zheng HSP, Wead HSPB, Zhang J, Li RC, Bolli R, Ping P. PKCepsilon activation induces dichotomous cardiac phenotypes and modulates PKCepsilon-RACK interactions and RACK expression. Am J Physiol Heart Circ Physiol. 2001;280:946-55.

10. Gibson TJ. RACK1 research - ships passing in the night? FEBS Lett. 2012; 586:2787-9.

11. Ron D, Adams DR, Baillie GS, Long A, O'Connor R, Kiely PA. RACK1 to the future-a historical perspective. Cell Commun Signal. 2013;11:53.

12. Gandin V, Senft D, Topisirovic I, Ronai ZA. RACK1 function in cell motility and protein synthesis. Genes Cancer. 2013;4(9-10):369-77.

13. Speth $C$, Laubinger S. RACK1 and the microRNA pathway: is it déjà-vu all over again? Plant Signal Behav. 2014;9:e27909.

14. Li JJ, Xie D. RACK1, a versatile hub in cancer. Oncogene. 2015;34:1890-8.

15. Corsini E, Galbiati V, Esser PR, Pinto A, Racchi M, Marinovich M, Martin SF, Galli CL. Role of PKC- $\beta$ in chemical allergen-induced CD86 expression and IL-8 release in THP-1 cells. Arch Toxicol. 2014;88:415-24.

16. Vermeulen A. Dehydroepiandrosterone sulfate and ageing. Ann NY Acad Sci. 1995;774:121-7.

17. Traish AM, Kang HP, Saad F, Guay AT. Dehydroepiandrosterone (DHEA)-a precursor steroid or an active hormone in human physiology. J Sex Med. 2011;8:2960-82.

18. Labrie F, Luu-The V, Labrie C, Simard J. DHEA and its transformation into androgens and estrogens in peripheral target tissues: intracrinology. Front Neuroendocrinol. 2001;22:185-212.

19. Webb SJ, Geoghegan TE, Prough RA, Michael Miller KK. The biological actions of dehydroepiandrosterone involves multiple receptors. Drug Metab Rev. 2006:38:89-116.

20. Bauer ME. Stress, glucocorticoids and ageing of the immune system. Stress. 2005;8:69-83.

21. Pinto A, Malacrida B, Oieni J, Serafini MM, Davin A, Galbiati V, Corsini E, Racchi M. DHEA modulates the effect of cortisol on RACK1 expression via interference with the splicing of the glucocorticoid receptor. $\mathrm{Br}$ J Pharmacol. 2015:172:2918-27.

22. Adler AJ, Danielsen M, Robins DM. Androgen-specific gene activation via a consensus glucocorticoid response element is determined by interaction with nonreceptor factors. Proc Natl Acad Sci U S A. 1992;89:11660-3.

23. Schoenmakers E, Verrijdt G, Peeters B, Verhoeven G, Rombauts W, Claessens $F$. Differences in DNA binding characteristics of the androgen and glucocorticoid receptors can determine hormone-specific responses. J Biol Chem. 2000:275:12290-7.

24. Livak KJ, Schmittgen TD. Analysis of relative gene expression data using real-time quantitative PCR and the 2(-Delta Delta C(T)) Method. Methods. 2001;25:402-8

25. Caruso D, Pesaresi M, Maschi O, Giatti S, Garcia-Segura LM, Melcangi RC. Effect of short-and long-term gonadectomy on neuroactive steroid levels in the central and peripheral nervous system of male and female rats. J Neuroendocrinol. 2010;22:1137-47.

26. Chanput W, Mes JJ, Wichers HJ. THP-1 cell line: an in vitro cell model for immune modulation approach. Int Immunopharmacol. 2014;23:37-45.

27. Buoso E, Lanni C, Molteni E, Rousset F, Corsini E, Racchi M. Opposing effects of cortisol and dehydroepiandrosterone on the expression of the receptor for activated C kinase 1: implications in immunosenescence. Exp Gerontol. 2011:46:877-83

28. Racchi M, Balduzzi C, Corsini E. Dehydroepiandrosterone (DHEA) and the aging brain: flipping a coin in the "fountain of youth". CNS Drug Rev. 2003;9:21-40.

29. Del Vecchio I, Zuccotti A, Pisano F, Canneva F, Lenzken SC, Rousset F, Corsini E, Govoni S, Racchi M. Functional mapping of the promoter region of the GNB2L1 human gene coding for RACK1 scaffold protein. Gene. 2009; 430:17-29.
30. Kaufman M, Pinsky L. The dissociation of testosterone- and 5 alphadihydrotestosterone-receptor complexes formed within cultured human genital skin fibroblasts. J Steroid Biochem. 1983;18:121-5.

31. Cadwallader AB, Rollins DE, Lim CS. Effect of anabolic-androgenic steroids and glucocorticoids on the kinetics of hAR and hGR nucleocytoplasmic translocation. Mol Pharm. 2010;7:689-98.

32. Lai JJ, Lai KP, Chuang KH, Chang P, Yu IC, Lin WJ, Chang C. Monocyte/ macrophage androgen receptor suppresses cutaneous wound healing in mice by enhancing local TNF-alpha expression. J Clin Invest. 2009;119:3739-51.

33. Chuang KH, Altuwaijri S, Li G, Lai JJ, Chu CY, Lai KP, Lin HY, Hsu JW, Keng P, Wu MC, Chang C. Neutropenia with impaired host defense against microbial infection in mice lacking androgen receptor. J Exp Med. 2009;206:1181-99.

34. Csaba G. Hormones in the immune system and their possible role. A critical review. Acta Microbiol Immunol Hung. 2014;61:241-60.

35. Trigunaite A, Dimo J, Jørgensen TN. Suppressive effects of androgens on the immune system. Cell Immunol. 2015;294:87-94.

\section{Submit your next manuscript to BioMed Central and we will help you at every step:}

- We accept pre-submission inquiries

- Our selector tool helps you to find the most relevant journal

- We provide round the clock customer support

- Convenient online submission

- Thorough peer review

- Inclusion in PubMed and all major indexing services

- Maximum visibility for your research

Submit your manuscript at www.biomedcentral.com/submit 